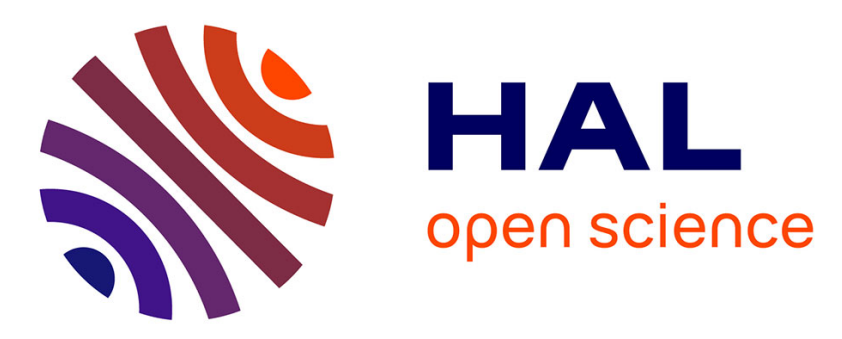

\title{
Chainlike Structure Formed in Iodine Monochloride Graphite Intercalation Compounds
}

Ferdinand Hof, Anthony Impellizzeri, Emmanuel Picheau, Xiaoyang Che, Alain Pénicaud, Christopher P Ewels

\section{- To cite this version:}

Ferdinand Hof, Anthony Impellizzeri, Emmanuel Picheau, Xiaoyang Che, Alain Pénicaud, et al.. Chainlike Structure Formed in Iodine Monochloride Graphite Intercalation Compounds. Journal of Physical Chemistry C, 2021, 125, pp.23383 - 23389. 10.1021/acs.jpcc.1c06303 . hal-03407819

\section{HAL Id: hal-03407819 https://hal.science/hal-03407819}

Submitted on 28 Oct 2021

HAL is a multi-disciplinary open access archive for the deposit and dissemination of scientific research documents, whether they are published or not. The documents may come from teaching and research institutions in France or abroad, or from public or private research centers.
L'archive ouverte pluridisciplinaire HAL, est destinée au dépôt et à la diffusion de documents scientifiques de niveau recherche, publiés ou non, émanant des établissements d'enseignement et de recherche français ou étrangers, des laboratoires publics ou privés. 


\section{Chain-Like Structure Formed in Iodine Monochloride Graphite Intercalation Compounds}

Ferdinand Hof, ${ }^{a, *}$ Anthony Impellizzeri, ${ }^{b}$ Emmanuel Picheau, ${ }^{a}$ Xiaoyang Che,${ }^{a, b}$ Alain Pénicaud, ${ }^{a}$ Christopher P. Ewels ${ }^{b, *}$

a. Univ. Bordeaux, CNRS, Centre de Recherche Paul Pascal, UMR5031, 33600 Pessac, France.

b. Université de Nantes, CNRS, Institut des Matériaux Jean Rouxel, F-44000, Nantes, France

Publié dans J. Phys. Chem. C, https://doi.org/10.1021/acs.jpcc.1c06303

ABSTRACT The structure of iodine monochloride (ICl) intercalated into graphite (GIC) has been revisited, and a stage 1 stoichiometry of about 10 carbon atoms to $1 \mathrm{ICl}$ molecule is determined. Intercalation proceeds readily at room temperature without additional energy input. A chain-like structure of alternating halogen species is proposed for the intercalation layer based on detailed Raman spectroscopic analysis and ab-initio calculations. Long range ordering is observed, reaching beyond one layer of carbon. Calculated density of states shows p-doping of the carbon layers upon intercalation, in agreement with Raman spectroscopic analysis. The intercalation is reversible upon heating and can be cycled several times. While Iodine chains have been observed in other nanocarbons, ICl is the first example of extended halogen chains intercalated in graphite. Indeed, intercalant phases of acceptor GICs have rarely been studied to date, and the current findings are potentially of interest in structural chemistry, material science, and solid-state batteries and other areas.

\section{Introduction}


Reversibly intercalating ions into host materials, especially graphite, is the essence of rockingchair type batteries. The most famous example and archetype is the Li-ion battery, commercialized in 1991, that still today represents the benchmark anode. ${ }^{1,2}$ Graphite is a unique host lattice due to its redox amphoteric character, forming graphite intercalation compounds (GICs) with both donor and acceptor compounds. ${ }^{3-11}$ These GICs exhibit fascinating properties and have been described as synthetic metals, due to their electrical behavior. Even superconductivity, albeit at low temperature, has been observed for some GICs. ${ }^{12,13}$ One of the most interesting aspects of GICs is that mostly defined ordering, so-called staging, is observed, where the distance between two adjacent carbon layers is well defined. ${ }^{14-16}$ Stage 1 is the simplest of all possible structures, and here each layer of carbon alternates with one layer of intercalant. In general, the intercalant is not randomly distributed but has the tendency to group together into domains. In case of lower intercalant concentration, this leads to multiple carbon layers without any intercalant, followed by a filled intercalant layer, and is conventionally called stage 2, stage 3 and so forth. This behavior is especially striking at very low intercalant concentrations and stable, crystallographically ordered stages of more than stage 8 have been described (8 layers of carbon followed by 1 layer of intercalant). ${ }^{4}$ The observation of stages has been explained by an interplay between long range cooperative interactions and electron transfer, either from or to the carbon layer. Both aspects favor the maximizing of the intercalant concentration in one domain. ${ }^{17,18}$ Although, the freedom of motion of the intercalant is reduced, a high diffusibility of the intercalant is commonly observed inside the intercalant layer, ${ }^{19,20}$ which explains the importance of GICs for modern batteries as mentioned above. ${ }^{21}$ Moreover, graphite intercalation compounds (GICs) are the basis for various liquid based exfoliation processes, including graphite oxide, superacid based methods, and graphenide solutions. ${ }^{22-30}$

A detailed understanding of the intercalant layer in GICs is made harder by the tendency to form domains, the difficulty distinguishing between kinetic and thermodynamic products, and diffusibility within the intercalant layer. ${ }^{31}$ In consequence, for the vast majority of GICs, the distance between nearest carbon layers is known in detail, whereas the position and structure of the intercalant molecules is unknown. Notable exceptions are mostly on the donor side of GICs for which detailed structural investigations have been performed, notably $\mathrm{KC}_{8}$, and lately $\mathrm{SrC}_{6}{ }^{32}$ On the acceptor side, the degree of complexity is much more pronounced, and knowledge of the structures formed in the intercalant layer is sparse. The best studied acceptor GIC is arguably 
$\mathrm{Br}_{2}$-GIC, where a maximum of stage 2 GICs can be reached and depending on the temperature, either a commensurable, incommensurable or liquid like structure in the intercalant layer is formed. ${ }^{33,34}$ In stark contrast to bromine, the other pure halogens, e.g. iodine do not readily intercalate. ${ }^{4}$ Thus, obtaining stage 1 compounds of halogen containing GICs, is not readily possible and synthetically challenging.

Polar heteroatomic halogen GICs such as iodine monochloride (ICl), although barely studied, have been known since the 1950s, and offer access to GICs at room temperature overcoming the above-mentioned difficulties. ${ }^{35-43}$ The stoichiometry of a stage-1 ICl GIC intercalation compound, i.e. consisting of alternating single layers of carbon and intercalant, has been suggested to be close to $10: 1$ in analogy with other acceptor intercalation compounds (e.g $\left.\mathrm{FeCl}_{3}\right) .^{44,45}$ Early structural analysis by Abe et al. ${ }^{45}$ reported an interlayer spacing of $7.14 \AA$. Moret et al. ${ }^{41}$ performed detailed crystallographic analysis and suggested that the stage 1 GIC exhibits an incommensurate structure with a large unit cell, proposing up to $9 \mathrm{ICl}$ molecules in the unit cell. ${ }^{46}$ Later extended X-Ray Absorption spectroscopy and Near Edge X-Ray absorption fine structure analysis (EXAFS) observed characteristic distances assigned to I-Cl and halogencarbon bonds. ${ }^{42}$ More recently, Jadaun et al..$^{38}$ used Density Functional Theory (DFT) to study a structure similar to the one reported by Tiedtke et al. ${ }^{47}$ However, despite this research effort, the structure of ICl-GICs is not entirely understood. Notably, the ordering and interaction of the ICl molecules inside the intercalant layer has not been described in detail. Furthermore, a detailed analysis of the electronic behavior has yet to be performed. In our manuscript, we discuss the synthesis of a stage 1 compound with a stoichiometry of 9.8 carbon atoms to 1 molecule of ICl (ICl-C..8 GIC) and its analysis by statistical Raman analysis, XRD analysis, and small angle Xray scattering experiments. The experimental results are corroborated by detailed ab-initio calculations, based on the analysis of various structural configurations and stoichiometries (ratio between ICl- $\mathrm{C}_{8.0}$ to ICl- $\mathrm{C}_{25.0}$ ). We find that ICl forms close-packed continuous chains within the intercalant layer. These chains have a "zig-zag" staggered sequence, where chlorine atoms flank iodine atoms in a quasi-linear arrangement. The calculated low frequency Raman stretch mode $\left(185 \mathrm{~cm}^{-1}\right)$ matches nicely with the observed experimental peak $\left(190.8 \pm 6.5 \mathrm{~cm}^{-1}, \kappa_{\mathrm{exc}}=532 \mathrm{~nm}\right)$, with calculated charge transfer from the carbon sheets to the intercalant. The intercalation process is highly reversible, as shown by temperature dependent SAXS measurements. 


\section{Method:}

In a heat-dried and argon-purged round-bottomed Schlenk flask (50 mL), $162.3 \mathrm{mg}(1.0 \mathrm{mmol})$ of liquid iodine monochloride (ICl) was placed. Afterwards, $120 \mathrm{mg}$ (10.0 mmol) graphite was added under argon and the flask is immediately sealed by a glass cap. Right after the addition of the graphite, all of the liquid ICl is spontaneously soaked up by the graphite. Afterwards, the asproduced graphite intercalation compound (GIC) is left to equilibrate for $48 \mathrm{~h}$ under constant pressure. Afterwards, the as-produced GIC was carefully transferred to another heat-dried and argon purged round-bottomed Schlenk flask $(50 \mathrm{~mL})$ and the stoichiometry was determined by weight uptake. The sample was placed under argon in a $80 \mathrm{~mm}, \varnothing 1.5 \mathrm{~mm}$ Lindemann capillary and sealed by a flame torch. This capillary was used for all physical characterization. More detailed physicochemical characterisation as well as computational detail can be found in ESI.

\section{Result and discussion}

Graphite intercalation compounds (GICs) have been prepared using iodine monochloride (ICl) as intercalant. The resulting ratio has been determined by weight uptake, giving a stoichiometry of 9.8 carbon atoms to 1 molecule of ICl (Scheme 1). The reaction is directly performed by reacting liquid ICl with graphite. The complete volume of liquid ICl is absorbed by graphite within several minutes, but at least 48 hours are needed for equilibration at room temperature. It is noteworthy that the intercalation is spontaneous and no stirring, heating or sonication is required.

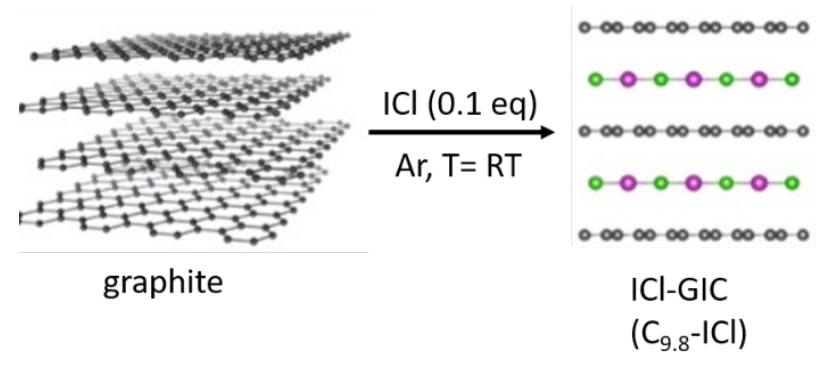

Scheme 1 Reaction scheme of the intercalation of ICl in graphite with a stoichiometry of 9.8 carbon atoms to 1 molecule of $\mathrm{ICl}\left(\mathrm{ICl}-\mathrm{C}_{9.8} \mathrm{GIC}\right)$ at room temperature under argon atmosphere. 
The sample was then sealed in Lindemann capillaries which subsequently have been used for all analysis. XRD analysis (see Figure 1 ) gives strong peaks at $25.64^{\circ}$ and $52.66^{\circ}$ which are assigned to (002) and (004) planes with an interlayer spacing $I_{c}=6.95 \AA$.

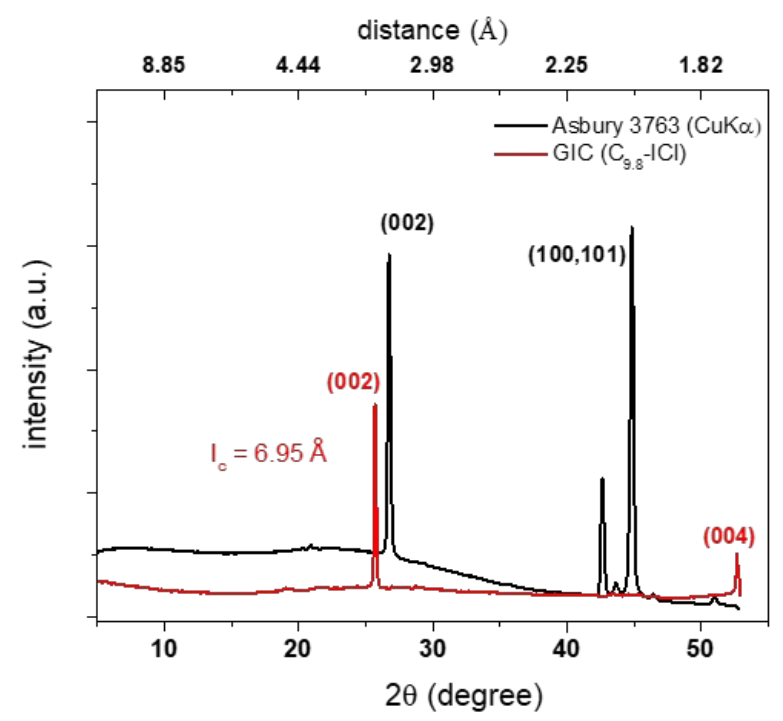

Figure 1: XRD patterns $(\mathrm{CuK} \alpha)$ of graphite intercalation compound containing ICl, stoichiometry of 9.8 carbon atoms to 1 molecule of ICl (red trace), in comparison to the starting graphite (black trace).

This observed interlayer spacing is slightly lower than $7.14 \AA$ reported by Abe et al. ${ }^{45}$ Interestingly, their data shows a (001) peak, at least an order-of-magnitude less intense than the (002) peak, not visible in our data. Temperature dependent x-ray scattering experiments confirm that only the (002) peak can be seen, which vanishes at $50^{\circ} \mathrm{C}$, and re-appears again upon cooling (Fig S1, supporting information). Furthermore, several peaks can be observed between $4.5 \AA$ and $5.0 \AA$, which similarly vanish upon heating and re-appear on cooling (Fig S1).

In donor GICs (e.g. $S_{C} C_{6}$ ), an $A \alpha A \beta$ stacking sequence $(A=$ carbon layers, $\alpha, \beta=$ intercalant layers) has been observed, ${ }^{32}$ and the (001) peak is absent due to extinction rules. The $\alpha, \beta$ of the intercalant layer highlights the long-range ordering present in GICs, where the ordering in one intercalant layer induces structural ordering in adjacent ones. For ICl- $\mathrm{C}_{9.8}$ GIC a similar structure with a $A \alpha A \beta$ stacking order is proposed. Structural elucidation is challenging based solely on experimental data, thus density functional theory (DFT) calculations were performed using the AIMPRO package, ${ }^{48-50}$ an approach previously successfully applied to other halogenated nanocarbons..$^{51,52}$ We address the choice of exchange-correlation functional and dispersion 
description further in supporting information, including benchmarking methods to experimentally measurable quantities (Table S1, S2, see also ESI). Different ICl structures were examined in both graphite and graphene systems, all of which show exothermic binding compared to isolated ICl. On graphene the most stable structure corresponds to a configuration of ICl perpendicular to the graphene plane, with iodine above a carbon atom (Fig. S2), similar to previous findings for $\mathrm{Br}_{2} \cdot{ }^{53}$ In contrast, when confined between two or more carbon layers, ICl adopts a configuration parallel to the graphene plane (Fig. S3).

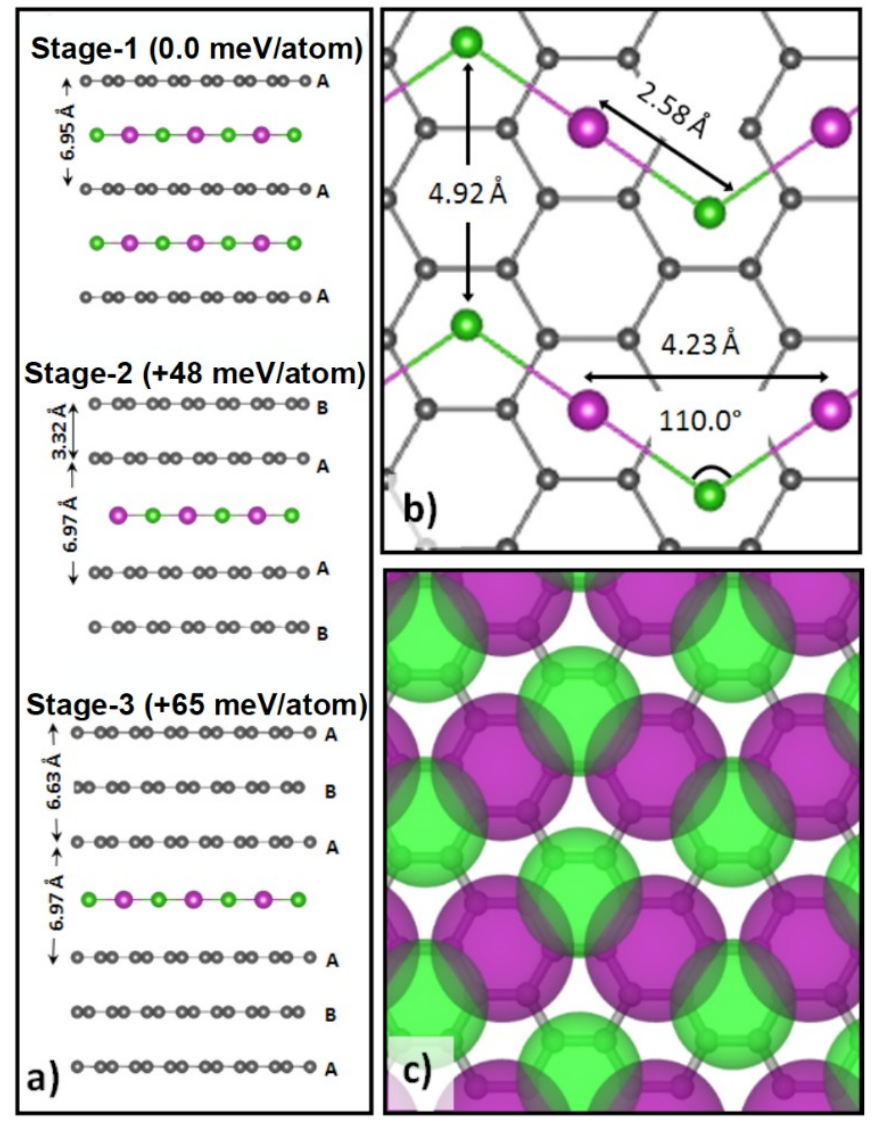

Figure 2: a) Optimized structures for Stage-n ICl-graphite, $n=1,3$. Carbon, Iodine and Chlorine atoms are in grey, purple and green, respectively. The relative binding energy per atom (eV/atom) is given compared to Stage-1, b) top view of the Stage-1 intercalant layer, exhibiting a chain like configuration, with characteristic distances in $\AA$, c) drawn with van der Waals radii of the halogen atoms.

A concentration dependant study was carried out for ICl-graphite from stage-1 to stage-3 (Figure 2), considering a range of molecular and extended configurations. The most stable 
intercalant structure is an alternating $\mathrm{Cl} / \mathrm{I}$ zig-zag chain-like configuration constructed from linear Cl-I-Cl units (Fig. 2b). This configuration is also favored in solid state $\mathrm{ICl},{ }^{54,55}$ and is due to interaction of $\sigma$-holes ${ }^{56}$ between neighbouring interhalogen molecules. All other configurations tested were considerably less stable (Fig S3). The angle in the chain was found to be $109.96^{\circ}$, about $20^{\circ}$ higher than in solid $\mathrm{ICl} .^{54,55}$ The Iodine atom sits above the centre of a graphite hexagon, similar to $\mathrm{Br}_{2}-\mathrm{GIC},{ }^{34,40}$ but the long $\mathrm{I}-\mathrm{Cl}$ bond forces the chlorine atoms to sit slightly off the hexagon centers. Inter-chain spacing is dictated by a superposition of the underlying graphene lattice symmetry with the maximum packing density allowed by the van der Waals radii of the halogen atoms (Fig. 2c). Bond lengths are remarkably similar for different stages. The I-Cl bond is $2.58 \AA$ (Fig. 2b), considerably larger than in solid ICl $(2.44 \AA)^{54,55}$ and close to early experimental EXAFS data by Krone et al.(2.54 $\AA) .{ }^{42}$ Other key distances include the $\mathrm{Cl}-\mathrm{Cl}$ interchain distance of $4.92 \AA$ and the Iodine-iodine intrachain distance of $4.23 \AA$ (Fig. 2b).The calculated graphite interlayer distance for Stage- 1 of $6.95 \AA$ matches exactly our experimental XRD value (see Figure 1). In agreement with the experiment, the calculations show an $\mathrm{A} \alpha \mathrm{A} \beta$ stacking sequence to be more stable than $\mathrm{A} \alpha \mathrm{A} \alpha$ (Fig S4). This allows chains on one layer to sit above "vacant" hexagons of the layer below, resulting in more uniform charge distribution in the carbon layers (see below). The calculated interlayer distances of $10.29 \AA$ for Stage-2, and $13.60 \AA$ for Stage-3, match well the experimental values reported by Abe et al. ${ }^{45}$ In these cases the grouped carbon layers preferentially adopt AB and ABA stacking respectively. Relative binding energies per atom (taking graphite and the ICl isolated molecule as reference states, Fig. 2(a)) show that Stage-1 is energetically favored over Stages-2 and 3. Corresponding absolute binding energies are $-110(-21),-61(-12)$, and $-44(-9) \mathrm{meV} /$ atom for Stages-1, 2, and 3, respectively, taking molecular $\mathrm{ICl}$ (isolated zig-zag $\mathrm{ICl}$ chain) as the reference state.

Statistical Raman analysis $\left(\lambda_{\text {exc }}=532,638,785 \mathrm{~nm}\right),{ }^{57}$ based on the fitting of 1681 individual spectra with Lorenzian functions (Figure 3) has been performed. Typical Raman spectra are depicted in Figure 3a, for the three excitation wavelengths used. The large background signal between $100-600 \mathrm{~cm}^{-1}$ at $785 \mathrm{~nm}$ excitation is arguably due to fluorescence. Several low frequency Raman peaks are observed, which vary both in peak position and intensity (see Fig. S5,S6, Tab. S4-6). Only one common low frequency peak has been observed at $190 \mathrm{~cm}^{-1}$ at $532 \mathrm{~nm}$ and $785 \mathrm{~nm}$ excitation wavelengths. The peak is not dispersive, and has an asymmetric lower frequency profile suggesting a close doublet. This correlates well with the chain model. 
Frequency calculations show a Raman active stretch mode at $180 \mathrm{~cm}^{-1}$ (symmetry A') (Fig 3a) which shows weak interchain coupling, splitting into modes at 179 and $185 \mathrm{~cm}^{-1}$ for the symmetric and antisymmetric coupling of neighbouring chains.
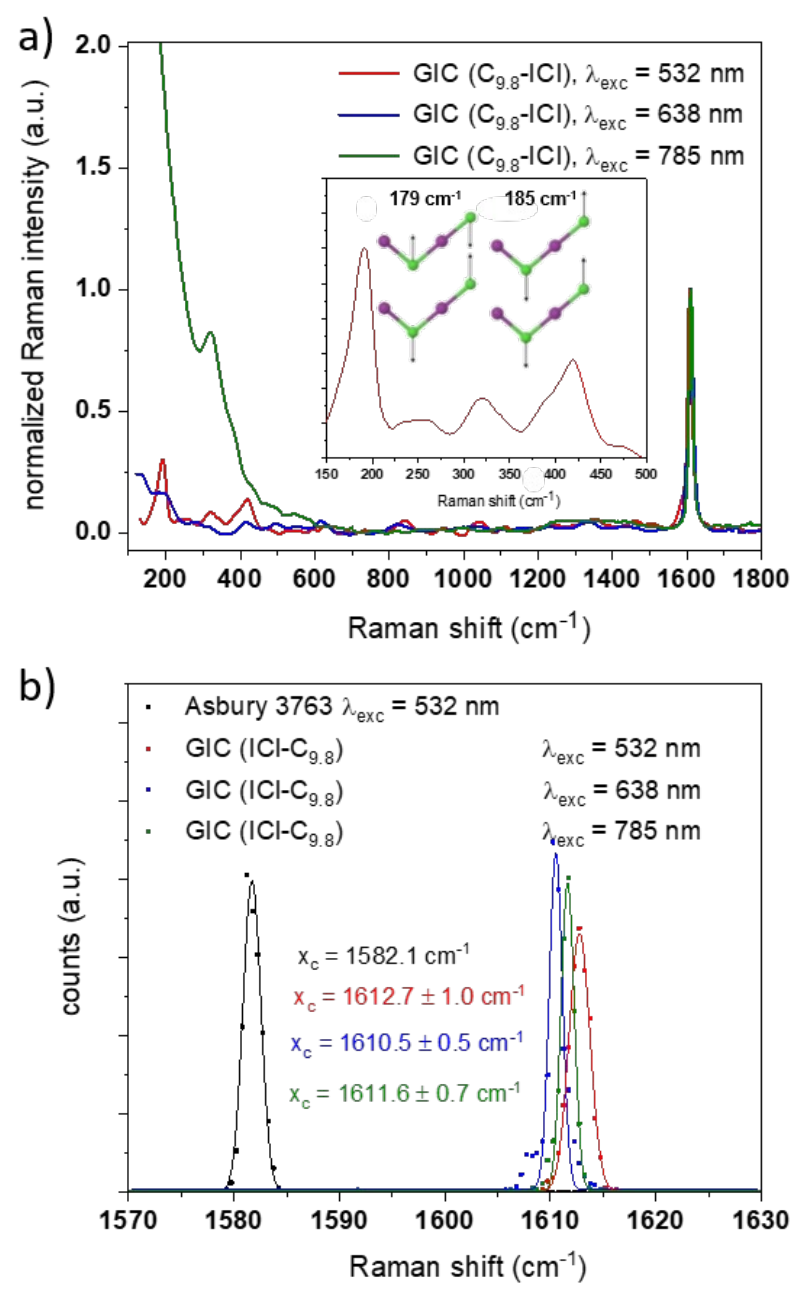

Figure 3: Statistical Raman spectroscopic analysis $\left(\lambda_{\mathrm{exc}}=532,638,785 \mathrm{~nm}\right)$ on the stage 1 GIC based on the analysis of 1681 spectra obtained over $10000 \mu^{2}$. a) typical Raman spectra, inset: zoom in low frequency modes. b) histogram of the peak position of the $\mathrm{E}_{2 \mathrm{G} 2}$ mode of the GIC in comparison to the $\mathrm{E}_{2 \mathrm{G}}$ peak position of the starting graphite, binning scale $0.25 \mathrm{~cm}^{-1}$.

The $\mathrm{E}_{2 \mathrm{G} 2}$ peak position in the GIC is determined from the individual fits and a histogram is constructed after frequency counting (Figure 3b). The obtained peak positions are $1612.7 \pm 1.0 \mathrm{~cm}^{-1} \quad\left(\lambda_{\mathrm{exc}}=532 \mathrm{~nm}\right), \quad 1610.5 \pm 0.5 \mathrm{~cm}^{-1} \quad\left(\lambda_{\text {exc }}=638 \mathrm{~nm}\right), \quad$ and $\quad 1611.6 \pm 0.7 \mathrm{~cm}^{-1}$ $\left(\lambda_{\text {exc }}=785 \mathrm{~nm}\right)$. The energy related to the $\mathrm{E}_{2 \mathrm{G} 2}$ peak is remarkably precise and the variation of the 
peak position over $10000 \mu \mathrm{m}^{2}$ is smaller $\left(\sim 1.0 \mathrm{~cm}^{-1}\right)$ than in the starting graphite $\left(2.4 \mathrm{~cm}^{-1}\right)$, highlighting the homogeneity of the sample. This peak sharpening can be understood through the isolation of individual carbon layers by the ICl, removing interlayer stacking and associated local stacking variations (including dislocations and stacking fault). The peak position is not dispersive in relation to the excitation energy (Fig S7). This peak is significantly upshifted from the raw graphite value of $1582.1 \pm 2.4 \mathrm{~cm}^{-1}\left(\lambda_{\mathrm{exc}}=532 \mathrm{~nm}\right)$, indicative of strong $p$-doping. The observation of a shift in Raman spectroscopy is typically explained by a change of the equilibrium lattice parameter and modification of the phonon dispersions. ${ }^{58,59}$ In electrochemically top-gated graphene transistors, a peak position of $1610 \mathrm{~cm}^{-1}$ is related to a Fermi level shift of about $0.65 \mathrm{eV}$.

The residue compound of the GIC was generated by evaporation of ICl from graphite inside a fume hood for 2 weeks. A statistical Raman analysis and the mean spectra for this compound are depicted in Fig S8. The spectroscopic features typically observed for graphite samples are present, and virtually identically to those of the starting material. The peak at $190 \mathrm{~cm}^{-1}$ indicates that traces of ICl remain, in analogy to other residue compounds e.g. $\mathrm{Br}_{2}$-GIC and $\mathrm{AlCl}_{3}$-GIC. Careful analysis of the peak positions (Fig S9) shows that the sample is successfully deintercalated, and peak position variations are identical to the starting material. The I(D)/I(G) ratio is commonly used to discuss defect density. For the residue compound it is centered at 0.088 (with a longer tail end, see Fig S10), a typical value for almost defect-free natural graphite. Those measurements corroborate the SAXS measurements, that show the intercalation of ICl into graphite is reversible and does not functionalize the carbon lattice.

Charge transfer has been observed for other intercalation compounds, both experimentally and theoretically. ${ }^{60,61}$ The $p$-doping effect caused by ICl intercalation, clearly visible in the Raman experiments, has been evaluated further in the DFT calculated electronic structure. Figure 4 shows the calculated band structures and projected density of states for AB-stacked graphite, and ICl intercalated Stages 1 to 3. In all cases the Fermi level is pinned to the bottom of the empty ICl states. The carbon zero density of states point, which for AB-stacked Graphite sits at the Fermi level, becomes increasingly upshifted, depopulating graphite bonding states and transferring charge to the ICl. This upshift is constant for Stages 2 and 3 at $0.31 \mathrm{eV}$, increasing to $0.47 \mathrm{eV}$ for Stage- 1 . This is consistent with the layer contact; graphene sheets only contact ICl on 
a single side in Stages-2 and 3, whereas they have ICl on both sides in Stage-1. This upshift is a clear signature of carbon $p$-doping and in agreement with the Raman spectroscopic experiments. The same calculated upshift $(0.47 \mathrm{eV})$ is also found in the case of ICl chains on the surface of graphene (Table S3). Further information can be seen in the Stage-3 PDOS, where the carbon PDOS has been broken down into two, the layers neighbouring ICl in black, and the central layer in brown. The central layer PDOS is much closer to that of the pristine AB-Graphite, indicating that coupling is local.

The same variations are reflected in the calculated Raman mode which varies as 1602/1606 (1586, 1604), 1604 (1604) and 1607 (1612) $\mathrm{cm}^{-1}$ for Stage 3, 2 and 1 respectively (experimental values in brackets for comparison, Figure S11). In stage 3 compounds, a split into two peaks $\left(\sim 1586 \mathrm{~cm}^{-1}\right.$, and $\left.1604 \mathrm{~cm}^{-1}\right)$ can be experimentally observed, with an intensity ratio of about 1:2. This reflects the different types of graphene layer present in a Stage-3 GIC. The lower frequency peak is more like conventional graphite and originates from the graphene layer in the middle that is not in contact with the $\mathrm{ICl}$ and relatively undoped, while the higher frequency peaks are those in contact with ICl. This splitting can indeed be seen in our calculations where the central layer frequency $1602 \mathrm{~cm}^{-1}$ is lower than that of the layers touching ICl, and is close to our calculated value of $1599 \mathrm{~cm}^{-1}$ for pristine AB-graphite in the same cell. In the stage-2 all carbon layers are in contact with $\mathrm{ICl}$, with an average lower concentration than in stage 1 . That the internal layers in intercalation compounds are almost graphite like is also observed for donor compounds, ${ }^{62}$ in solid state $\mathrm{C}^{13}$-NMR measurements of $\mathrm{Br}_{2}$-GIC intercalation compound, ${ }^{60}$ and is in line with the discussion of Fischer about the electronic properties of GICs in the framework of one-electron theory. ${ }^{61}$ Funning now to the carbon-related bands in the band structures. While AB-graphite shows conventional parabolic bands at the Fermi level, Stage-1 shows linear crossing with a Dirac point as that of graphene (albeit with a Fermi level shift), while Stage-2 and Stage-3 show symmetry offset crossing points (Stage-2) and overlaid Dirac cones and parabolic bands (Stage3) respectively. This confirms that an ICl layer fully decouples interaction between its two neighbouring carbon layers. 

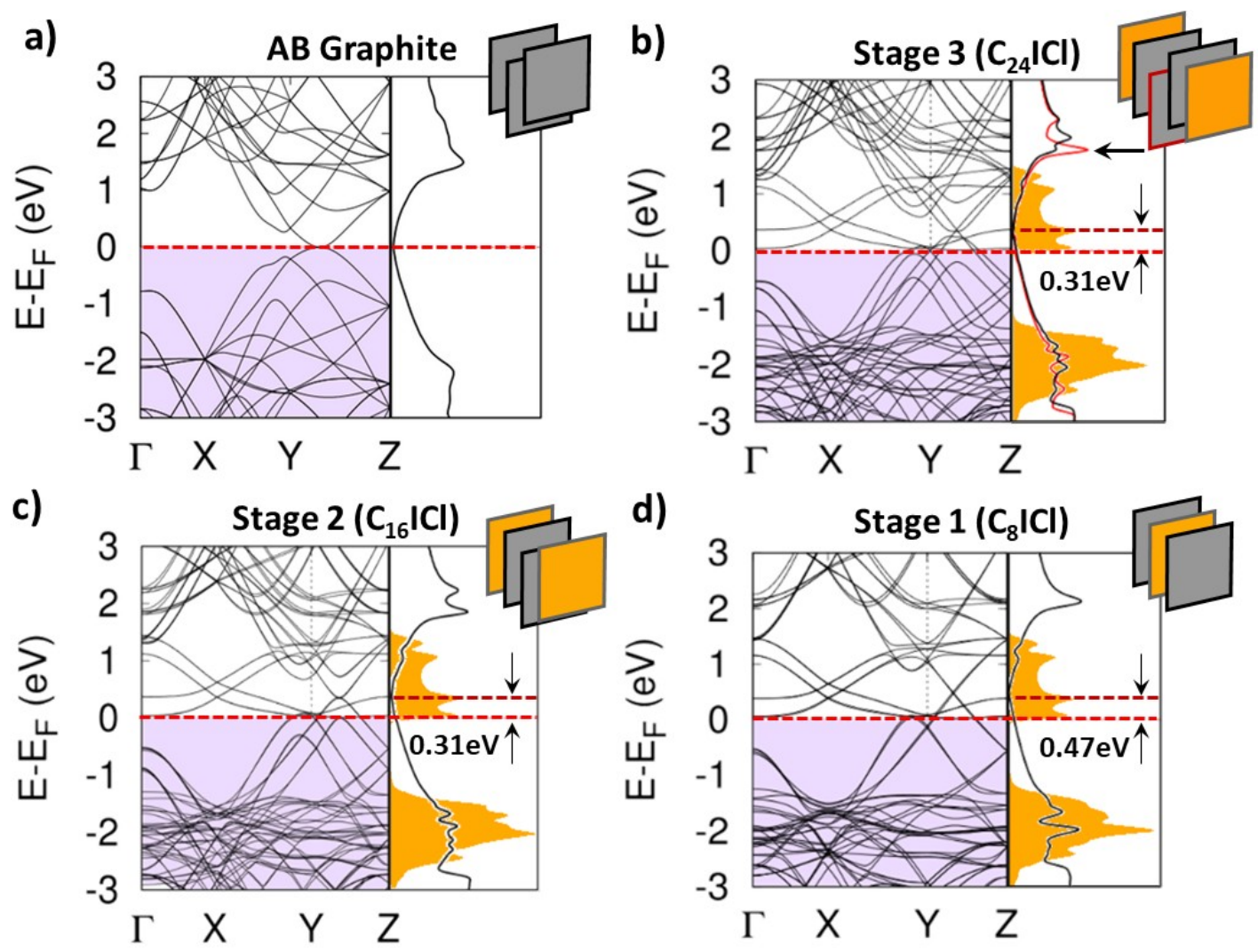

Figure 4 Electronic band structure and projected density of states (PDOS) of a) AB-stacked graphite, b) Stage 3 ICl-GIC (C24ICl), c) Stage 2 ICl-GIC (C16ICl) and d) Stage 1, ICl-GIC (C8ICl). Occupation is illustrated by the blue area and the Fermi level is set to $0 \mathrm{eV}$. Density of states to the right of each band structure is projected onto halogen atoms (orange area), neighbouring carbon layers (black curve), and intermediate carbon layer in brown for Stage-3. p-doping effect is visible in the downshift of the Fermi level of the graphite states. Vertical offset of the grey carbon layers in the schematics indicate $\mathrm{AB}$ and $\mathrm{ABC}$-type stacking.

\section{Conclusion}

Iodine monochloride graphite stage 1 intercalation compound has been synthesized and its electronic and vibrational properties have been studied in detail by physicochemical characterization as well as ab-initio methods. A GIC forms spontaneously at room temperature upon simple exposure of graphite to iodine monochloride. In this stage 1 compound, the observation of solely the (002), (004) patterns is compatible with a unit cell containing at least 2 layers of intercalant and 2 layers of carbon. Calculations indicate that the structure of the intercalant layer consists of close packed chains of iodine monochloride, iodine sitting above hexagon sites with chlorine occupying the exterior sites of the zigzag slightly displaced from 
hexagon centres. The double layer symmetry is due to offset of alternating ICl layers perpendicular to the chain direction, giving an $\mathrm{A} \alpha \mathrm{A} \beta$ stacking sequence. The calculated frequency of those chains is in good agreement with experimental Raman data. Both, the observation of long-range order and the formation of chains, show a significant ordering present for this GIC. The intercalation/deintercalation process can be cycled several times, demonstrating the reversibility of the phase transfer from a quasi- crystalline solid-state structure towards a non-correlated phase. P-doing upon intercalation is observed for all investigated stages. This detailed understanding of an intercalant phase of an acceptor GICs is highly interesting for the field of structural chemistry as well as material science and might provide anchor points for further studies for solid-state batteries and energy applications amongst others.

\section{ASSOCIATED CONTENT}

Supporting Information (ESI) Detailed Raman spectroscopy, SAXS, as well as computational details is available as PDF free of charge.

\section{AUTHOR INFORMATION}

\section{Corresponding Author}

Email: Ferdinand.Hof@u-bordeaux.fr (Ferdinand Hof)

Email: Chris.Ewels@cnrs-imn.fr (Christopher P.Ewels)

\section{Author Contributions}

FH conceived the project. FH, EP performed the experimental synthesis. AI, XC, CPE, performed the theoretical work. All authors contributed to the interpretation of the data and writing of the manuscript.

\section{Conflicts of interest}

The authors declare no competing interests 


\section{ACKNOWLEDGMENT}

FH and XC acknowledge funding from CNRS (Momentum 2017). We thank the CCIPL (centre de calculs intensif pays de la loire) where many of the calculations were performed. AI, EP, CPE, and AP acknowledge the ANR Project ANR-16-CE24-0008-01 "EdgeFiller". AI and CPE acknowledge the ANR Project ANR-20-CE08-0026 “OPIFCat”.

\section{REFERENCES}

(1) Li, Y.; Lu, Y.; Adelhelm, P.; Titirici, M.-M.; Hu, Y.-S. Intercalation Chemistry of Graphite: Alkali Metal Ions and Beyond. Chem. Soc. Rev. 2019, 48, 4655-4687.

(2) Jian, Z.; Luo, W.; Ji, X. Carbon Electrodes for K-Ion Batteries. J. Am. Chem. Soc. 2015, 137, 11566-11569.

(3) Fredenhagen, K. Die Bindung von Kalium Durch Kohlenstoff. J. Inorg. Gen. Chem. 1926, 158, 249-263.

(4) Levy, F. Intercalated Layered Materials; Lévy, F., Ed.; Springer Netherlands: Dordrecht, 1979; Vol. 1.

(5) Hennig, G. R. Interstitial Compounds of Graphite. In Progress in Inorganic Chemistry; 1959; Vol. 1, pp 125-205.

(6) Stumpp, E. The Intercalation of Metal Chlorides and Bromides into Graphite. Mater. Sci. Eng. 1977, 31, 53-59.

(7) Nixon, D. E.; Parry, G. S. Formation and Structure of the Potassium Graphites. J. Phys. D. Appl. Phys. 1968, 1, 303. 
(8) Guerard, D.; Herold, A. Intercalation of Lithium into Graphite and Other Carbons. Carbon 1975, 13, 337-345.

(9) Rüdorff, W. Graphite Intercalation Compounds (GIC). In Modern Problems in Condensed Matter Sciences; 1988; pp 197-321.

(10) Rüdorff, W.; Schulze, E. Über Alkaligraphitverbindungen. Zeitschrift für Anorg. und Allg. Chemie 1954, 277, 156-171.

(11) Rybin, M. G.; Islamova, V. R.; Obraztsova, E. A.; Obraztsova, E. D. Modification of Graphene Electronic Properties via Controllable Gas-Phase Doping with Copper Chloride. Appl. Phys. Lett. 2018, 112, 033107.

(12) Weller, T. E.; Ellerby, M.; Saxena, S. S.; Smith, R. P.; Skipper, N. T. Superconductivity in the Intercalated Graphite Compounds C6Yb and C6Ca. Nat. Phys. 2005, 1, 39-41.

(13) Csányi, G.; Littlewood, P. B.; Nevidomskyy, A. H.; Pickard, C. J.; Simons, B. D. The Role of the Interlayer State in the Electronic Structure of Superconducting Graphite Intercalated Compounds. Nat. Phys. 2005, 1, 42-45.

(14) Millman, S. E.; Kirczenow, G. Origin of Simple Staging in Graphite Intercalation Compounds. Phys. Rev. B 1982, 26, 2310-2313.

(15) Safran, S. A.; Hamann, D. R. Electrostatic Interactions and Staging in Graphite Intercalation Compounds. Phys. Rev. B 1980, 22, 606-612.

(16) Safran, S. A. Phase Diagrams for Staged Intercalation Compounds. Phys. Rev. Lett. 1980, 44, 937-940. 
(17) Dahn, J. R.; Dahn, D. C.; Haering, R. R. Elastic Energy and Staging in Intercalation Compounds. Solid State Commun. 1982, 42, 179-183.

(18) Safran, S. A.; Hamann, D. R. Long-Range Elastic Interactions and Staging in Graphite Intercalation Compounds. Phys. Rev. Lett. 1979, 42, 1410-1413.

(19) Lauginie, P.; Estrade, H.; Conard, J.; Guérard, D.; Lagrange, P.; El Makrini, M. Graphite Lamellar Compounds EPR Studies. Phys. B+C 1980, 99, 514-520.

(20) Conard, J.; Estrade-Szwarckopf, H.; Lauginie, P.; Hermann, G. Magnetic Resonance in Graphite Intercalated Compounds. In L. Pietronero et al. (eds.), Physics of Intercalation Compounds; 1981; pp 264-273.

(21) Manthiram, A. A Reflection on Lithium-Ion Battery Cathode Chemistry. Nat. Commun. 2020, 11, 1-9.

(22) Behabtu, N.; Lomeda, J. R.; Green, M. J.; Higginbotham, A. L.; Sinitskii, A.; Kosynkin, D. V.; Tsentalovich, D.; Parra-Vasquez, A. N. G.; Schmidt, J.; Kesselman, E.; et al. Spontaneous High-Concentration Dispersions and Liquid Crystals of Graphene. Nat. Nanotechnol. 2010, 5, 406-411.

(23) Chung, D. D. L. A Review of Exfoliated Graphite. J. Mater. Sci. 2016, 51, 554-568.

(24) Vallés, C.; Drummond, C.; Saadaoui, H.; Furtado, C. A.; He, M.; Roubeau, O.; Ortolani, L.; Monthioux, M.; Pénicaud, A. Solutions of Negatively Charged Graphene Sheets and Ribbons. J. Am. Chem. Soc. 2008, 130, 15802-15804.

(25) Chua, C. K.; Pumera, M. Chemical Reduction of Graphene Oxide: A Synthetic Chemistry 
Viewpoint. Chem. Soc. Rev. 2014, 43, 291-312.

(26) Eigler, S.; Hirsch, A. Chemistry with Graphene and Graphene Oxide - Challenges for Synthetic Chemists. Angew. Chemie - Int. Ed. 2014, 53, 7720-7738.

(27) Hummers, W. S.; Offeman, R. E. Preparation of Graphitic Oxide. J. Am. Chem. Soc. 1958, 80, 1339-1339.

(28) Thiele, H. Über Die Quellung von Graphit. Zeitschrift für Anorg. und Allg. Chemie 1932, 206, 407-415.

(29) Dimiev, A. M.; Tour, J. M. Mechanism of Graphene Oxide Formation. ACS Nano 2014, 8, 3060-3068.

(30) Catheline, A.; Vallés, C.; Drummond, C.; Ortolani, L.; Morandi, V.; Marcaccio, M.; Iurlo, M.; Paolucci, F.; Pénicaud, A. Graphene Solutions. Chem. Commun. (Camb). 2011, 47, 5470-5472.

(31) Safran, S. A. Domain Growth of Degenerate Phases. Phys. Rev. Lett. 1981, 46, 15811584.

(32) El Hajj, I.; Speyer, L.; Cahen, S.; Lagrange, P.; Medjahdi, G.; Hérold, C. Crystal Structure of First Stage Strontium-Graphite Intercalation Compound. Carbon 2020, 168, 732-736.

(33) Simon, C.; Rosenman, I.; Batallan, F.; Pepy, G.; Lauter, H. Neutron Diffraction on Bromine Intercalated in Graphite. Synth. Met. 1988, 23, 147-153.

(34) Sasa, T.; Takahashi, Y.; Mukaibo, T. Crystal Structure of Graphite Bromine Lamellar Compounds. Carbon 1971, 9, 407-416. 
(35) Croft, R. New Molecular Compounds of the Layer Lattice Type. I. New Molecular Compounds of Graphite. Aust. J. Chem. 1956, 9, 184.

(36) Rüdorff, W.; Sils, V.; Zeller, R. Über Das Verhalten von Graphit Gegenüber Jodmonochlorid Und Chromylchlorid. Zeitschrift für Anorg. und Allg. Chemie 1956, 283, 299-303.

(37) Hennig, G. The Properties of the Interstitial Compounds of Graphite. III. The Electrical Properties of the Halogen Compounds of Graphite. J. Chem. Phys. 1952, 20, 1443-1447.

(38) Jadaun, P.; Movva, H. C. P.; Register, L. F.; Banerjee, S. K. Theory and Synthesis of Bilayer Graphene Intercalated with ICl and IBr for Low Power Device Applications. J. Appl. Phys. 2013, 114.

(39) Heerschap, M.; Delavignette, P.; Amelinckx, S. Electron Microscope Study of Interlamellar Compounds of Graphite with Bromine, Iodine Monochloride and Ferric Chloride. Carbon 1964, 1, 235-243.

(40) Eeles, W. T, T. J. A. The Crystal Structure of Graphite-Bromine Compounds. Proc. $R$. Soc. London. Ser. A. Math. Phys. Sci. 1965, 283, 179-193.

(41) Moret, R.; Rousseaux, F.; Furdin, G.; Herold, A. Single-Crystal X-Ray Study of GraphiteBr and Graphite-Icl Intercalation Compounds. Synth. Met. 1983, 7, 289-295.

(42) Krone, W.; Wortmann, G.; Kaindl, G. Intercalate Structure in Halogen-Intercalated Graphite. Synth. Met. 1989, 29, 247-252.

(43) Culik, J. S.; Chung, D. D. L. Calorimetric Study of the Order-Disorder Transformations in 
Graphite-Halogens. Mater. Sci. Eng. 1979, 37, 213-221.

(44) Abe, T.; Mizutani, Y.; Shinoda, N.; Ihara, E.; Asano, M.; Harada, T.; Inaba, M.; Ogumi, Z. Debye-Waller Factors of FeCl3- and ICl-Graphite Intercalation Compounds. Carbon 1995, 33, 1789-1793.

(45) Abe, T.; Mizutani, Y.; Shinoda, N.; Asano, M.; Harada, T.; Murakami, M. Debye-Waller Factors of ICl-Graphite Intercalation Compounds Prepared from Natural Graphite Flakes and Graphitized Polyimide Films. J. Phys. Chem. Solids 1996, 57, 783-786.

(46) Ghosh, D.; Gangwar, R.; Chung, D. D. L. Superlattice Ordering in Graphite-IC1 Single Crystals and Fibers. Carbon 1984, 22, 325-333.

(47) Tiedtke, M.; Wortmann, G. The Chemical Nature of Interhalogen Molecules in ICL and IBr-Graphite From127I-And129I-Mössbauer Spectroscopy. Hyperfine Interact. 1990, 53, 419-424.

(48) Briddon, P. R.; Jones, R. LDA Calculations Using a Basis of Gaussian Orbitals. Phys. Status Solidi Basic Res. 2000, 217, 131-171.

(49) Rayson, M. J.; Briddon, P. R. Rapid Iterative Method for Electronic-Structure Eigenproblems Using Localised Basis Functions. Comput. Phys. Commun. 2008, 178, $128-134$.

(50) Rayson, M. J.; Briddon, P. R. Highly Efficient Method for Kohn-Sham Density Functional Calculations of 500-10000 Atom Systems. Phys. Rev. B - Condens. Matter Mater. Phys. 2009, 80, 1-11. 
(51) Sedelnikova, O. V.; Ewels, C. P.; Pinakov, D. V.; Chekhova, G. N.; Flahaut, E.; Okotrub, A. V.; Bulusheva, L. G. Bromine Polycondensation in Pristine and Fluorinated Graphitic Carbons. Nanoscale 2019, 11, 15298-15306.

(52) Rybkovskiy, D. V.; Impellizzeri, A.; Obraztsova, E. D.; Ewels, C. P. Polyiodide Structures in Thin Single-Walled Carbon Nanotubes: A Large-Scale Density-Functional Study. Carbon 2019, 142, 123-130.

(53) Yaya, A.; Ewels, C. P.; Suarez-Martinez, I.; Wagner, P.; Lefrant, S.; Okotrub, A.; Bulusheva, L.; Briddon, P. R. Bromination of Graphene and Graphite. Phys. Rev. B 2011, 83, 045411.

(54) van der Heide, J.; Vos, A.; Wiebenga, E. H. The Crystal Structure of $\alpha$-ICl. Acta Crystallogr. 1956, 9, 274-277.

(55) Carpenter, G. B.; Richards, S. M. The Crystal Structure of $\beta$-Iodine Monochloride. Acta Crystallogr. 1962, 15, 360-364.

(56) Cavallo, G.; Metrangolo, P.; Milani, R.; Pilati, T.; Priimagi, A.; Resnati, G.; Terraneo, G. The Halogen Bond. Chem. Rev. 2016, 116, 2478-2601.

(57) Hof, F.; Bosch, S.; Englert, J. M. J. M.; Hauke, F.; Hirsch, A. Statistical Raman Spectroscopy: A Method for the Characterization of Covalently Functionalized SingleWalled Carbon Nanotubes. Angew. Chemie Int. Ed. 2012, 51, 11727-11730.

Das, A.; Pisana, S.; Chakraborty, B.; Piscanec, S.; Saha, S. K.; Waghmare, U. V; Novoselov, K. S.; Krishnamurthy, H. R.; Geim, A. K.; Ferrari, A. C.; et al. Monitoring 
Dopants by Raman Scattering in an Electrochemically Top-Gated Graphene Transistor. Nat. Nanotechnol. 2008, 3, 1-6.

(59) Pietronero, L.; Strässler, S. Bond-Length Change as a Tool to Determine Charge Transfer and Electron-Phonon Coupling in Graphite Intercalation Compounds. Phys. Rev. Lett. 1981, 47, 593-596.

(60) Conard, J.; Gutierrez-Le Brun, M.; Lauginie, P.; Estrade-Szwarckopf, H.; Hermann, G. High Field 13C N.M.R. in Lamellar Compounds of Graphite and a Model of "in Plane Localized” Densities of States. Synth. Met. 1980, 2, 227-236.

(61) Fischer, J. E. Graphite Intercalation Compounds: Electronic Properties and Their Correlation with Chemistry. Phys. B+C 1980, 99, 383-394.

(62) Wang, Y.; Puech, P.; Gerber, I.; Pénicaud, A. Resonant Raman Scattering of Graphite Intercalation Compounds KC 8 , KC 24 , and KC 36. J. Raman Spectrosc. 2014, 45, 219 223. 